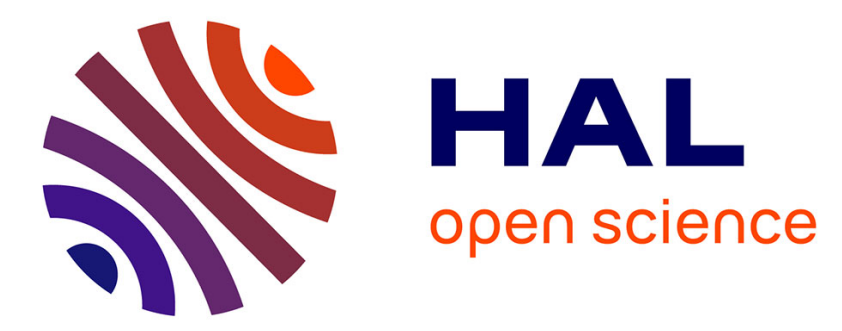

\title{
Sparse-based estimators improvement in case of basis mismatch
}

Stéphanie Bernhardt, Remy Boyer, Sylvie Marcos, Pascal Larzabal

\section{To cite this version:}

Stéphanie Bernhardt, Remy Boyer, Sylvie Marcos, Pascal Larzabal. Sparse-based estimators improvement in case of basis mismatch. IEEE 6th International Workshop on Computational Advances in Multi-Sensor Adaptive Processing (CAMSAP 2015), Dec 2015, Cancùn, Mexico. hal-01245384

\section{HAL Id: hal-01245384 \\ https://hal.science/hal-01245384}

Submitted on 3 Mar 2020

HAL is a multi-disciplinary open access archive for the deposit and dissemination of scientific research documents, whether they are published or not. The documents may come from teaching and research institutions in France or abroad, or from public or private research centers.
L'archive ouverte pluridisciplinaire HAL, est destinée au dépôt et à la diffusion de documents scientifiques de niveau recherche, publiés ou non, émanant des établissements d'enseignement et de recherche français ou étrangers, des laboratoires publics ou privés. 


\section{Sparse-Based Estimators Improvement in case of Basis Mismatch}

\author{
Stéphanie Bernhardt, Rémy Boyer and Sylvie Marcos \\ L2S \\ Université Paris-Sud (UPS), CNRS, CentraleSupelec \\ Gif-Sur-Yvette, France \\ \{stephanie.bernhardt, remy.boyer, sylvie.marcos\}@1ss.supelec.com
}

\author{
Pascal Larzabal \\ SATIE \\ ENS Cachan \\ Cachan, France \\ pascal.larzabal@satie.ens-cachan.fr
}

\begin{abstract}
Compressed sensing theory promises to sample sparse signals using a limited number of samples. It also resolves the problem of under-determined systems of linear equations when the unknown vector is sparse. Those promising applications induced a growing interest for this field in the past decade. In compressed sensing, the sparse signal estimation is performed using the knowledge of the dictionary used to sample the signal. However, dictionary mismatch often occurs in practical applications, in which case the estimation algorithm uses an uncertain dictionary knowledge. This mismatch introduces an estimation bias even when the noise is low and the support (i.e. location of non-zero amplitudes) is perfectly estimated. In this paper we consider that the dictionary suffers from a structured mismatch, this type of error being of particular interest in sparse estimation applications. We propose the Bias-Correction Estimator (BiCE) post-processing step which enhances the non-zero amplitude estimation of any sparse-based estimator in the presence of a structured dictionary mismatch. We give the theoretical Bayesian Mean Square Error of the proposed estimator and show its statistical efficiency in the low noise variance regime.
\end{abstract}

Keywords-Compressed Sensing with Uncertainty, Basis Mismatch, Oblique projectors

\section{INTRODUCTION}

Many natural signals are sparse either naturally, or after projecting them over an appropriate basis. Taking advantage of this property, compressed sensing allows to sample those signals with much less samples than requested by Shannon's sampling theory. To do so, the signals are sampled using a measurement matrix and reconstructed using a sparse-promoting criterion [1], [2], [3]. Many efficient algorithms efficiently handle this problem as for instance the OMP [4], CoSAMP [5], BPDN and LASSO, ...

However in practical context, the dictionary may not be perfectly known [6]. This is the case for example in digital data hiding applications [7], [8] or compressed wireless communication [9], [10], where the dictionary needs to be transmitted such that the receiver can decode the signal. Dictionary mismatch can also occur if the basis is constructed using a discretization of a given parameter space, in which case the receiver may not know exactly this discretization and reconstruct a slightly shifted grid which results in a dictionary

This work was supported by the European Commission in the framework of the FP7 Network of Excellence in Wireless COMmunications NEWCOM\# (Grant agreement no. 318306), and by the two following projects: ANR MAGELLAN and MI-TITAN. mismatch at the estimation step. The effect of dictionary mismatch has been studied in [11], [12], it has been shown that even for a small mismatch error, the accuracy of the amplitude estimation is drastically limited even for a low noise variance and a perfectly estimated support [13].

To mitigate the considered problem, we propose a new estimation scheme, called the Bias-Correction Estimator (BiCE), which is a post-processing adapted to any sparse-based estimator. The BiCE allows to efficiently mitigate the Basis mismatch effect for an acceptable extra computational cost.

\section{STRUCTURED MISMATCH BASIS PROBLEM}

\section{A. Compressed Sensing modelization}

Let $\boldsymbol{\Psi}$ be a $N \times K$ sensing/measurement matrix with $N<$ $K$. In compressed sensing (CS), the $N \times 1$ noisy measurements $\mathbf{y}$ [1], [3] are given by:

$$
\mathbf{y}=\mathbf{\Psi} \mathbf{s}+\mathbf{n}
$$

in which $\mathbf{s} \stackrel{\text { def. }}{=} \boldsymbol{\Phi} \boldsymbol{\theta}$ where matrix $\mathbf{\Phi}$ is a $K \times K$ orthonormal dictionary and $\boldsymbol{\theta}$ is a $L$-sparse amplitude vector. We assume that the support, $\mathcal{S}$, is of cardinality $L$, and that the non-zero amplitudes in vector $\boldsymbol{\theta}$, denoted by $\boldsymbol{\theta}_{\mathcal{S}}$, are i.i.d. with zero mean and a variance of $\sigma_{\theta}^{2}$. Finally, the noise $\mathbf{n}$ is a centered circular Gaussian white noise of unknown covariance $\sigma^{2} \mathbf{I}$. The CS model (1) is given by

$$
\mathbf{y}=\boldsymbol{\Psi} \boldsymbol{\Phi} \boldsymbol{\theta}+\mathbf{n} \stackrel{\text { def. }}{=} \mathbf{H}_{\mathcal{S}} \boldsymbol{\theta}_{\mathcal{S}}+\mathbf{n}
$$

where $\mathbf{H}_{\mathcal{S}}$ is the $L$ columns extracted from the $N \times K$ dictionary matrix $\mathbf{H}$ corresponding to support $\mathcal{S}$.

Ideally, if $\mathbf{H}$ satisfies the Restricted Isometry Property (RIP) [3], [2] and is perfectly known, it is possible to estimate $\boldsymbol{\theta}$ using the convex $l_{1}$ norm sparse promoting constraint. However, in practical scenario only a mismatched version of the dictionary, denoted by $\tilde{\mathbf{H}}$, can be available. We are interested in this scenario, especially in the case where the mismatched error is structured.

\section{B. Structured dictionary mismatch}

Dictionary based estimation is one of the common way to take profit of compressed sensing theory: in sparse estimation the sparsity promoting basis $\Phi$ is created by a discretization of a known continuous function/kernel $\phi(t)$, function which 
is dependent of the signal model of the specific application of interest (Radar [14], DoA estimation [15], ...) . Let $T_{S}$ be the sampling period, we have $[\boldsymbol{\Phi}]_{k k^{\prime}}=\left.\phi(t)\right|_{t=\tau_{k^{\prime}}-k T_{S}}$ where $1 \leq k, k^{\prime} \leq K$ and $\tau=\left[\tau_{1}, \cdots, \tau_{K}\right]^{T}$ is the given dicretization of the parameter set. The most common case is the uniform discretization of the grid with $\tau_{k^{\prime}}=k^{\prime} T_{S}$. Using this type of dictionary, the mismatch on the basis matrix $\tilde{\boldsymbol{\Phi}}$ comes from a uncertain discretization of function $\phi(t)$, defined as $\tilde{\tau}=\left[\tilde{\tau}_{1}, \cdots, \tilde{\tau}_{K}\right]^{T}$ where $\tilde{\tau}_{k^{\prime}}=\tau_{k^{\prime}}+e_{k^{\prime}}$. For a sufficiently small mismatched error, the first-order Taylor approximation of the basis $\tilde{\boldsymbol{\Phi}}$ is given by

$$
\begin{aligned}
{[\tilde{\mathbf{\Phi}}]_{k k^{\prime}} } & =\left.\phi(t)\right|_{t=\tilde{\tau}_{k^{\prime}}-T_{S} k} \\
& =\left.\phi(t)\right|_{t=\tau_{k^{\prime}}-T_{S} k}+\left.\frac{\partial \phi(t)}{\partial t}\right|_{t=\tau_{k^{\prime}}-T_{S} k} e_{k^{\prime}}+O\left(e_{k^{\prime}}^{2}\right) .
\end{aligned}
$$

An equivalent formalism for $k^{\prime} \in \mathcal{S}$ is $\tilde{\boldsymbol{\Phi}}_{\mathcal{S}} \approx \boldsymbol{\Phi}_{\mathcal{S}}+$ $\dot{\boldsymbol{\Phi}}_{\mathcal{S}} \operatorname{diag}\left(\mathbf{e}_{\mathcal{S}}\right)$ where $\left[\dot{\boldsymbol{\Phi}}_{\mathcal{S}}\right]_{k k^{\prime}}=\left.\frac{\partial \phi(t)}{\partial t}\right|_{t=\tau_{k^{\prime}}-T_{S} k}$ and $\mathbf{e}_{\mathcal{S}}=$ $\left[\ldots e_{k^{\prime}}, \ldots\right]^{T}$ where $k^{\prime} \in \mathcal{S}$. In term of the dictionary, we have the following approximation:

$\tilde{\mathbf{H}}_{\mathcal{S}}=\boldsymbol{\Psi} \tilde{\boldsymbol{\Phi}}_{\mathcal{S}} \approx \boldsymbol{\Psi} \boldsymbol{\Phi}_{\mathcal{S}}+\boldsymbol{\Psi} \dot{\boldsymbol{\Phi}}_{\mathcal{S}} \operatorname{diag}\left(\mathbf{e}_{\mathcal{S}}\right)=\mathbf{H}_{\mathcal{S}}+\dot{\mathbf{H}}_{\mathcal{S}} \operatorname{diag}\left(\mathbf{e}_{\mathcal{S}}\right)$.

This structured dictionary mismatch modelization has been largely adopted in the literature (see [16] for instance and the references therein).

\section{BICE FOR A STRUCTURED MISMATCHED DICTIONARY}

Here after, the $\mathrm{BiCE}$ is introduced and its theoretical accuracy is derived.

\section{A. Description of the BiCE}

The BiCE is designed as an efficient and computational attractive post-processing which improves standard sparse recovery estimators. The general description of the $\mathrm{BiCE}$ is given on Fig. 1. This algorithm is based on the estimation of vector $\hat{\boldsymbol{\theta}}$ computed by any standard sparse estimators, denoted on Fig. 1 by $\mathcal{A}(\cdot)$, to perform a corrected amplitude vector estimation. This is done through an ad-hoc oblique projector based only on the knowledge of the corrupted dictionary, $\tilde{\mathbf{H}}_{\hat{\mathcal{S}}}$, and its firstorder derivative, $\dot{\tilde{\mathbf{H}}}_{\hat{\mathcal{S}}}$. We recall that in the Basis mismatch problem, the knowledge of the initial uncorrupted dictionary is not available. As demonstrated in the sequel, our strategy allows to mitigate the dictionary mismatch with a limited additional computational workload.

\section{B. Analysis of the accuracy of the BiCE}

Result 3.1: In the regime where the support set has been estimated without error and for sufficiently small $\sigma^{2} \sigma_{e}^{2}$, the conditional bias vector and the BMSE can be approximated according to

$$
\begin{aligned}
& \operatorname{Bias}\left(\hat{\boldsymbol{\theta}}_{\mathcal{S}} \mid \boldsymbol{\theta}_{\mathcal{S}}\right) \approx \sigma_{e}^{2} \mathbf{H}_{\mathcal{S}}^{\dagger} \mathbf{E}_{\mathbf{H}_{\mathcal{S}} \dot{\mathbf{H}}_{\mathcal{S}}} \ddot{\mathbf{H}}_{\mathcal{S}} \boldsymbol{\theta}_{\mathcal{S}}, \\
& \operatorname{BMSE}\left(\hat{\overline{\boldsymbol{\theta}}}_{\mathcal{S}}\right) \approx \sigma^{2} F_{\mathcal{S}}+\sigma_{\theta}^{2} m_{e}^{4} \bar{F}_{\mathcal{S}}
\end{aligned}
$$

Data: $\mathbf{y}, \tilde{\mathbf{H}}, L, \phi(t)$

Result: $\operatorname{BiCE}-\mathcal{A}(\mathbf{y}, \tilde{\mathbf{H}}, L, \phi(t))$ estimate: $\hat{\overline{\boldsymbol{\theta}}}_{\hat{\mathcal{S}}}$

1) Run the sparse-based estimator ie.,

$$
\mathcal{A}(\mathbf{y}, \tilde{\mathbf{H}}, L, \phi(t)) \rightarrow \hat{\boldsymbol{\theta}} \text {. }
$$

2) Compute $\dot{\tilde{\mathbf{H}}}=\boldsymbol{\Psi} \dot{\tilde{\boldsymbol{\Phi}}}$ where $[\dot{\tilde{\boldsymbol{\Phi}}}]_{k k^{\prime}}=$ $\left.\frac{\partial \phi(t)}{\partial t}\right|_{t=\tilde{\tau}_{k^{\prime}}-T_{S} k}$.

3) From $\hat{\boldsymbol{\theta}}$ compute $\hat{\mathcal{S}}$.

4) Using the support set estimation $\hat{\mathcal{S}}$, compute $\tilde{\mathbf{H}}_{\hat{\mathcal{S}}}$ and $\dot{\tilde{\mathbf{H}}}_{\hat{\mathcal{S}}}$.

5) Compute the oblique projector [17] for range space $\left\langle\tilde{\mathbf{H}}_{\hat{\mathcal{S}}}\right\rangle$ and null space $\left\langle\dot{\tilde{\mathbf{H}}}_{\hat{\mathcal{S}}}\right\rangle$ :

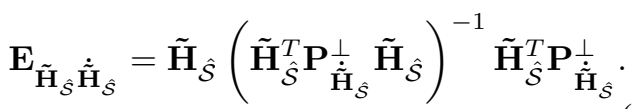

6) Finally, compute

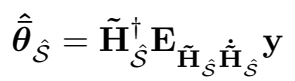

where $\dagger$ stands for the Moore-Penrose pseudoinverse.

\section{Algorithm 1: $\operatorname{BiCE}-\mathcal{A}(\mathbf{y}, \tilde{\mathbf{H}}, L, \phi(t))$}

where $m_{e}^{4}=\mathbb{E}\left(e_{i}^{4}\right)$ stands for the 4 -th order moment and

$$
\begin{aligned}
& F_{\mathcal{S}}=\operatorname{Tr}\left[\left(\mathbf{H}_{\hat{\mathcal{S}}}^{T} \mathbf{P}_{\dot{\mathbf{H}}_{\hat{\mathcal{S}}}^{\perp}}^{\perp} \mathbf{H}_{\hat{\mathcal{S}}}\right)^{-1}\right], \\
& \bar{F}_{\mathcal{S}}=\operatorname{Tr}\left[\ddot{\mathbf{H}}_{\mathcal{S}}^{T} \mathbf{P}_{\dot{\mathbf{H}}_{\mathcal{S}}}^{\perp} \mathbf{H}_{\mathcal{S}}\left(\mathbf{H}_{\mathcal{S}}^{T} \mathbf{P}_{\dot{\mathbf{H}}_{\mathcal{S}}}^{\perp} \mathbf{H}_{\mathcal{S}}\right)^{-2} \mathbf{H}_{\mathcal{S}}^{T} \mathbf{P}_{\dot{\mathbf{H}}_{\mathcal{S}}}^{\perp} \ddot{\mathbf{H}}_{\mathcal{S}}\right] .
\end{aligned}
$$

Proof: Due to the lack of space, only a sketch of the proof is provided here. The aim is to derive $\hat{\overline{\boldsymbol{\theta}}}_{\mathcal{S}}-\boldsymbol{\theta}_{\mathcal{S}}$. To do this, the fist step is to use $\dot{\tilde{\mathbf{H}}} \approx \dot{\mathbf{H}}+\ddot{\mathbf{H}} \operatorname{diag}(\mathbf{e})$ in measurement y. The second step will be to exploit a first-order Taylor approximation of the orthogonal projector $\mathbf{P}_{\dot{\tilde{\mathbf{H}}}_{\hat{\mathcal{S}}}}^{\perp}$ [18]. After some derivations, the bias vector and the BMSE are given by (7) and (8).

Thanks to Result 3.1., we can give the following important remark.

Remark 3.2: For the scenario where there is no mismatched error $\left(\sigma_{e}^{2} \rightarrow 0\right)$, we have:

- The BiCE is conditionally unbiased.

- The BMSE of the BiCE converges toward $\sigma^{2} \operatorname{Tr}\left[\left(\mathbf{H}_{\hat{\mathcal{S}}}^{T} \mathbf{P}_{\dot{\mathbf{H}}}^{\perp} \mathbf{H}_{\hat{\mathcal{S}}}\right)^{-1}\right]$. According to [19], this expression is in fact the Expected Cramér-Rao Bound (ECRB) for the projected observation $\mathbf{y}$ onto the subspace $\left\langle\dot{\mathbf{H}}_{\mathcal{S}}\right\rangle^{\perp}$.

Remark 3.3: In the low noise variance regime $\left(\sigma^{2} \rightarrow 0\right)$, the order of magnitude of the BMSE of the BiCE is given by the 4-th order moment of the mismatched error. To illustrate this property, recall that the 4-th order moment for an error 


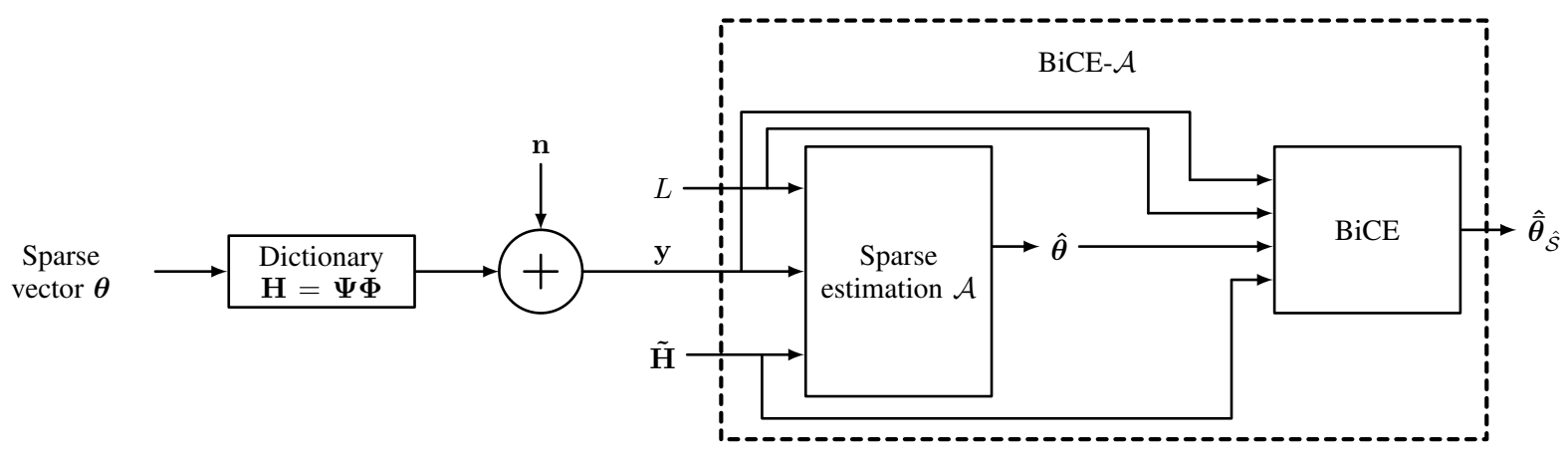

Fig. 1. Description of the BiCE- $\mathcal{A}$. Note that algorithm $\mathcal{A}(\cdot)$ stands for any sparse-based estimators.

following a Gaussian random variable of variance $\sigma_{e}$ is $3 \sigma_{e}^{4}$ which is rather small compared to the error without correction which is of order of $\sigma_{e}^{2}$. This key property of the BiCE is illustrated in the simulation part.

\section{Evaluation of the dominating computational cost in Flops}

In this section, the dominating computational cost of the $\mathrm{BiCE}$ is given and compared to the OMP computational cost. The OMP complexity is in $O(L N K)$ [20] for large $K$. The OMP is known to have a relatively low computational cost with respect to other sparse-based estimators.

The BiCE involves as a first step, the computation of a $(N-L)$ rank orthogonal projector, $\mathbf{P}_{\dot{\mathbf{H}}_{S}}^{\perp}$ which can be evaluated at the cost $O((N-L) N L)$ which is the cost of a reduced-rank SVD. The second step is the projection $\mathbf{Z}=\mathbf{P}_{\hat{\tilde{\mathbf{H}}}_{\mathcal{S}}}^{\perp} \tilde{\mathbf{H}}_{\mathcal{S}}$ which can be evaluated in $O\left(N^{2} L\right)$ and finally, the last step is the resolution of an ordinary LS criterion given by $\mathbf{Z}^{T} \mathbf{Z} \hat{\overline{\boldsymbol{\theta}}}_{\mathcal{S}}=\mathbf{Z} \mathbf{y}$ which is evaluated in $O\left(N L^{2}\right)$. So, we can conclude that the dominating cost of the BiCE is $O\left(N^{2} L\right)$. As $N \ll K$ by assumption, we can conclude that the BiCE dominating computational cost is much lower than the dominating cost of the OMP.

\section{APPLICATION TO NON-BANDLIMITED SIGNAL SAMPLING}

\section{A. Sampling of a stream of pulses}

To illustrate the potential improvements due to the BiCE post-processing, we consider the sampling of a nonbandlimited signal constituted by a stream of $L$ weighted and delayed pulses [21] given by $x(t)=\sum_{l \in \mathcal{S}} \theta_{l} \delta\left(t-\tau_{l}\right)$ where $\tau_{l}$ and $\theta_{l}$ are the unknown time-delay and amplitude of the $l$-th pulse, respectively and $\delta(t)$ is the Dirac delta function. The sample sequence is given by

$$
c_{k}=\sum_{l \in \mathcal{S}} \theta_{l} \phi\left(\tau_{l}-(k-1) T_{S}\right)+n_{k}, \quad 1 \leq k \leq K
$$

where $T_{S}$ is the sampling interval, $\phi(t)$ is chosen to be the Sinc sampling Kernel [21] and $n_{k}$ is a real discrete white Gaussian noise having zero-mean and a variance of $\sigma^{2}$. In the sequel, the basis $K \times K$ matrix is computed according to $[\boldsymbol{\Phi}]_{k k^{\prime}}=$ $\phi\left(\frac{\tau_{k^{\prime}}}{T_{S}}-(k-1)\right)$.

Interestingly and to the best of our knowledge, the sampling of non-bandlimited signals has never been presented in the CS framework, i.e. when the $K$-length sample sequence is randomly mapped onto a size-compressed $N$-length signal, denoted by $y_{n}$. We think that the CS theory is well adapted to the considered sampling problem. Consequently, the initial sample sequence $\mathbf{c}=\left[c_{1} \cdots c_{K}\right]^{T}$ is "compressed" according to $\mathbf{y}=\mathbf{\Psi} \mathbf{c}$ where $\boldsymbol{\Psi}$ is a "fat" $N \times K$ random matrix. To do so, the non-bandlimited signal sampling problem follows the CS model given in (1).

\section{B. Simulation results}

Two popular sparse-based estimators, namely, the OMP [4] and the CoSaMP [5], are considered in this simulation part to estimate the time-delays and the amplitudes based on the compressed measurements $\mathbf{y}$. We apply the BiCE for each algorithms and we name the enhanced version of these algorithms by BiCE-OMP and BiCE-CoSaMP. As a benchmark, we compare the BMSE measurements obtained by Monte Carlo simulations with the "oracle" ECRB [22] given by $\mathbb{E}\left\|\boldsymbol{\theta}_{\mathcal{S}}-\hat{\boldsymbol{\theta}}_{\mathcal{S}}\right\|^{2} \geq \operatorname{ECRB}=\sigma^{2} \operatorname{Tr}\left[\left(\mathbf{H}_{\mathcal{S}}^{T} \mathbf{H}_{\mathcal{S}}\right)^{-1}\right]$. Term "oracle" means that the dictionary is known and for the true support $\mathcal{S}$. Thus, this Bayesian lower bound gives the lowest accuracy that can be reached and will be compared with the theoretical BMSE of the BiCE given in (8). The SNR in the Bayesian context is defined by $\mathbb{E}\left(\left\|\boldsymbol{\theta}_{\mathcal{S}}\right\|^{2}\right) / \mathbb{E}\left(\|\mathbf{n}\|^{2}\right)$.

On Fig.2, the ideal scenario without mismatch error is considered. We can see that the OMP and the CoSaMP estimators are statistically efficient (i.e. reach the ECRB) when the noise variance is small enough. Note that the BiCE-OMP and BiCE-CoSaMP schemes do not modify the "behavior" of the OMP and CoSaMP algorithms in case of null mismatch error. It was crucial to check this fact to show that the proposed solutions can be used in any situations. On Fig.3, the mismatch error variance is fixed to $\sigma_{e}^{2}=1 e-3$. In this case, the OMP and the CoSaMP estimators show a "saturated" BMSE in the low noise variance regime and even if the support is perfectly estimated. We can also note that the BiCE strategy is efficient for the two tested sparse-based estimators since the BMSE of BiCE-OMP and BiCE-CoSaMP now reach the ECRB. Also, note that the simulated BMSE for the BiCE-OMP and the BiCE-CoSaMP match the theoretical BMSE given in (8) in the low noise variance regime.

\section{Conclusions}

Compressed Sensing with uncertain knowledge of the model is an important challenge since this scenario is realis- 


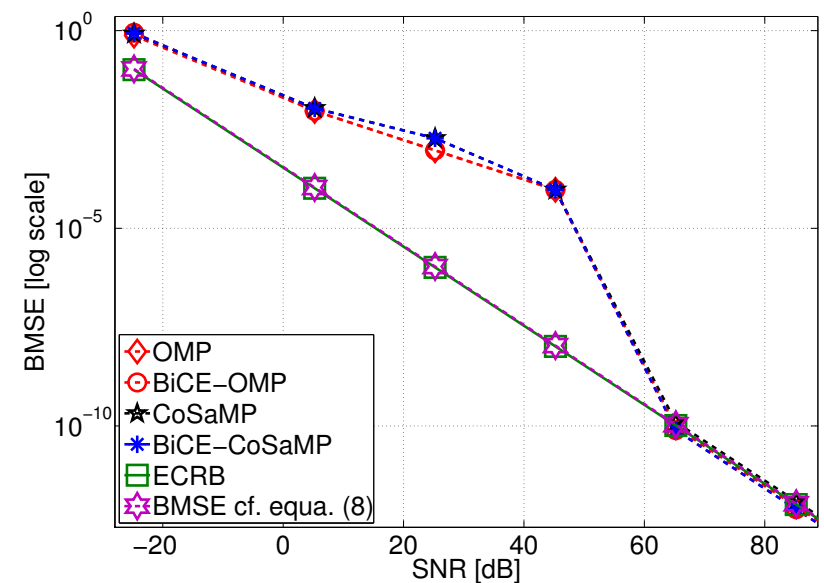

Fig. 2. BMSE $V s$. the SNR [dB] with $N=200, K=500, L=10$, $\sigma_{e}^{2}=0$

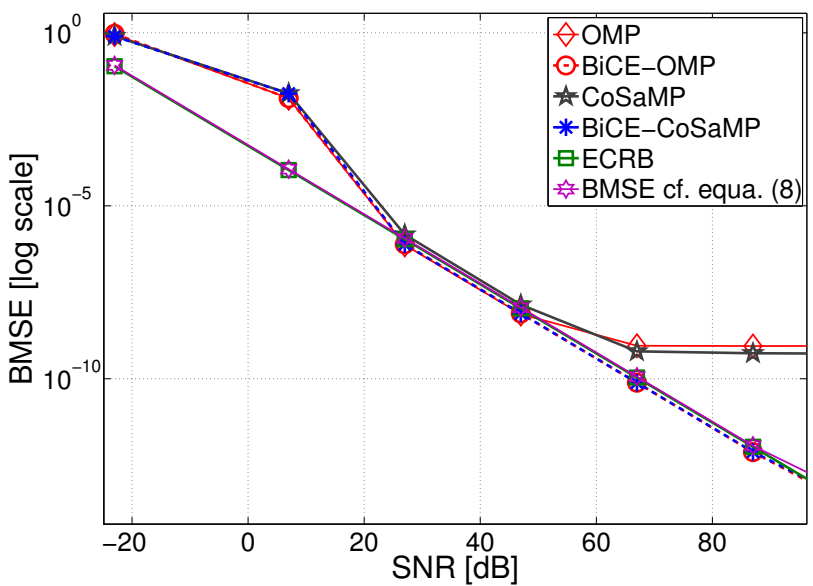

Fig. 3. BMSE Vs. the SNR [dB] with $N=300, K=1000, L=10$, $\sigma_{e}^{2}=1 e-3$

tic in operating context. Unfortunately, standard sparse-based estimators suffer from a limited estimation accuracy in the low noise variance regime and even if the support is perfectly estimated. In this paper, we proposed a general enhancement strategy called Bias Correction Estimator (BiCE) to improve the estimation of a sparse vector in presence of a dictionary mismatch. The BiCE is an extra/post layer estimation refinement which can be used for any sparse-based estimator. We derived the theoretical BMSE of the BiCE and illustrated by numerical simulations the improvement that we can hope. Specifically, when the sparse-based estimators suffer from a BMSE limitation, the combination between these schemes with the BiCE allows to obtain enhancement estimation schemes reaching the oracle ECRB which is the lowest estimation accuracy that we can expect. As a by product, the BiCE has a low computational complexity cost.

\section{REFERENCES}

[1] R. Baraniuk, "Compressive sensing [lecture notes]," Signal Processing Magazine, IEEE, vol. 24, no. 4, pp. 118-121, 2007.

[2] D. L. Donoho, "Compressed sensing," Information Theory, IEEE Transactions on, vol. 52, no. 4, pp. 1289-1306, 2006.
[3] E. J. Candes and T. Tao, "Decoding by linear programming," Information Theory, IEEE Transactions on, vol. 51, no. 12, pp. 4203-4215, 2005.

[4] Y. C. Pati, R. Rezaiifar, Y. C. Pati, and P. S. Krishnaprasad, "Orthogonal matching pursuit: Recursive function approximation with applications to wavelet decomposition," pp. 40-44, 1993.

[5] D. Needell and R. Vershynin, "Signal recovery from incomplete and inaccurate measurements via regularized orthogonal matching pursuit," Selected Topics in Signal Processing, IEEE Journal of, vol. 4, no. 2, pp. 310-316, 2010.

[6] M. Rosenbaum and A. B. Tsybakov, "Sparse recovery under matrix uncertainty," The Annals of Statistics, vol. 38, no. 5, pp. 2620-2651, 2010.

[7] I. J. Cox, M. L. Miller, and A. L. McKellips, "Watermarking as communications with side information," Proceedings of the IEEE, vol. 87, no. 7, pp. 1127-1141, 1999.

[8] C. Delpha, S. Hijazi, and R. Boyer, "A compressive sensing based quantized watermarking scheme with statistical transparency constraint," in Digital-Forensics and Watermarking, ser. Lecture Notes in Computer Science, Y. Q. Shi, H.-J. Kim, and F. Prez-Gonzlez, Eds. Springer Berlin Heidelberg, 2014, pp. 409-422.

[9] K. Hayashi, M. Nagahara, and T. Tanaka, "A user's guide to compressed sensing for communications systems," IEICE transactions on communications, vol. 96, no. 3, pp. 685-712, 2013.

[10] W. Bajwa, J. Haupt, A. Sayeed, and R. Nowak, "Compressive wireless sensing," in Proceedings of the 5th international conference on Information processing in sensor networks. ACM, 2006, pp. 134-142.

[11] Y. Chi, L. Scharf, A. Pezeshki, and A. Calderbank, "Sensitivity to basis mismatch in compressed sensing," Signal Processing, IEEE Transactions on, vol. 59, no. 5, pp. 2182-2195, 2011.

[12] D. Chae, P. Sadeghi, and R. Kennedy, "Effects of basis-mismatch in compressive sampling of continuous sinusoidal signals," in Future Computer and Communication (ICFCC), 2010 2nd International Conference on, vol. 2, 2010, pp. V2-739-V2-743.

[13] S. Bernhardt, R. Boyer, B. Zhang, S. Marcos, and P. Larzabal, "Performance analysis for sparse based biased estimator: Application to line spectra analysis," in Sensor Array and Multichannel Signal Processing Workshop (SAM), invited paper, 2014 IEEE 8th. IEEE, 2014, pp. 365-368.

[14] M. Herman and T. Strohmer, "High-resolution radar via compressed sensing," Signal Processing, IEEE Transactions on, vol. 57, no. 6, pp. 2275-2284, June 2009.

[15] D. Malioutov, M. Cetin, and A. Willsky, "A sparse signal reconstruction perspective for source localization with sensor arrays," Signal Processing, IEEE Transactions on, vol. 53, no. 8, pp. 3010-3022, 2005.

[16] H. Zhu, G. Leus, and G. Giannakis, "Sparsity-cognizant total leastsquares for perturbed compressive sampling," Signal Processing, IEEE Transactions on, vol. 59, no. 5, pp. 2002-2016, 2011.

[17] R. T. Behrens and L. L. Scharf, "Signal processing applications of oblique projection operators," Signal Processing, IEEE Transactions on, vol. 42, no. 6, pp. 1413-1424, 1994.

[18] H. Krim, P. Forster, and J. G. Proakis, "Operator approach to performance analysis of root-music and root-min-norm," Signal Processing, IEEE Transactions on, vol. 40, no. 7, pp. 1687-1696, 1992.

[19] R. Boyer, "Oblique projection for source estimation in a competitive environment: algorithm and statistical analysis," Signal Processing, vol. 89, no. 12, pp. 2547-2554, 2009.

[20] J. Wang, S. Kwon, and B. Shim, "Generalized orthogonal matching pursuit," Signal Processing, IEEE Transactions on, vol. 60, no. 12, pp. 6202-6216, 2012.

[21] M. Vetterli, P. Marziliano, and T. Blu, "Sampling signals with finite rate of innovation," Signal Processing, IEEE Transactions on, vol. 50, no. 6, pp. 1417-1428, 2002.

[22] Z. Ben-Haim and Y. Eldar, "A lower bound on the bayesian mse based on the optimal bias function," Information Theory, IEEE Transactions on, vol. 55, no. 11, pp. 5179-5196, 2009. 